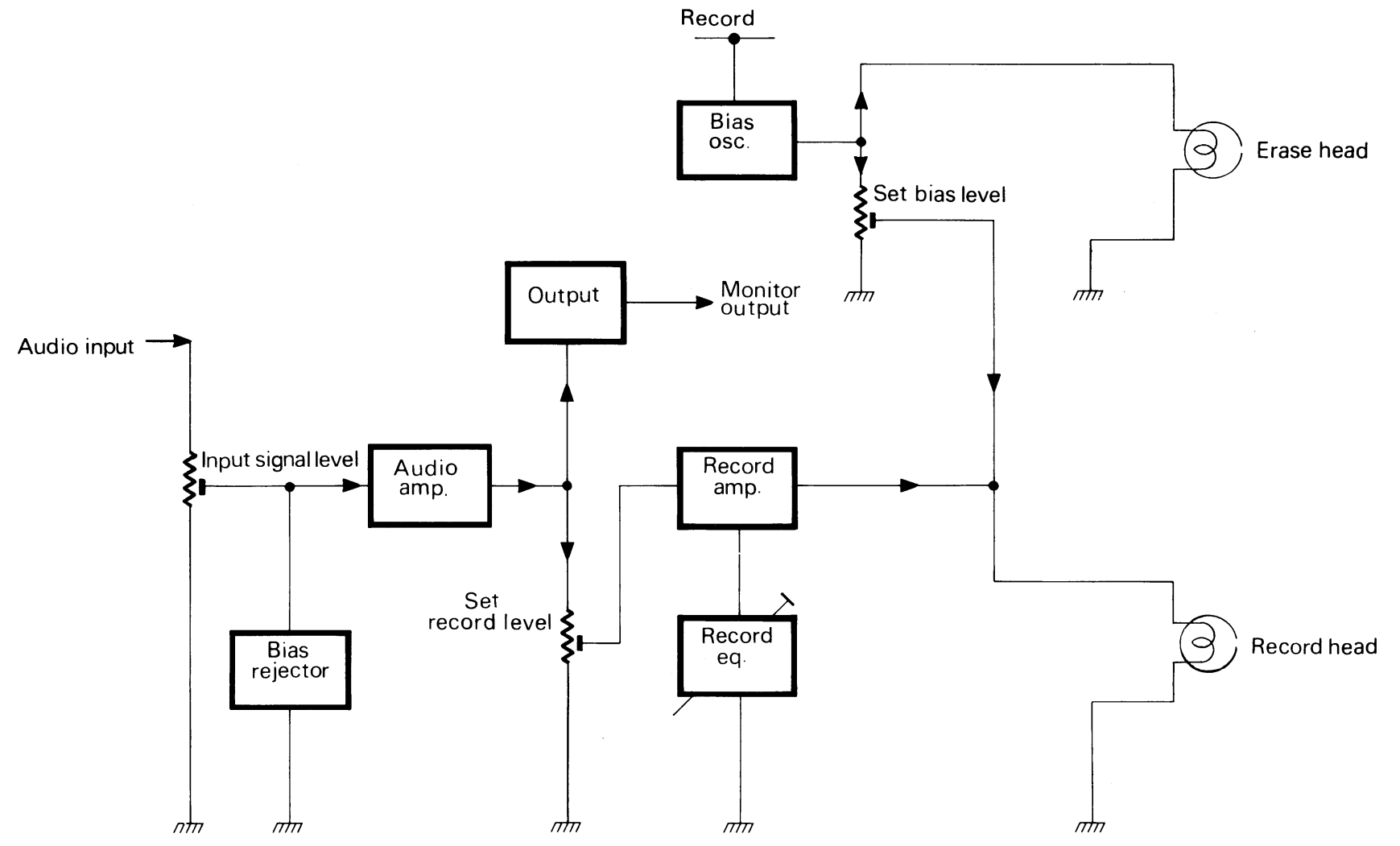

\section{Basic record system}

The diagram above shows a basic record system. Although the audio input signal has not been through a circuit where bias is present, the bias oscillator radiates a lot of energy which may be picked up in the audio path. For this reason there is a bias signal rejector before the audio amplifier. Signal from the top of the record level control from the audio amplifier is amplified and fed to an output socket. This provides an input for a monitor, thereby enabling the record signal to be heard if required.

The record equalisation circuit is frequency selective and operates (usually) by selective negative feedback.

\section{Basic playback system}

The audio signal off-tape is fed to a pre-amplifier which also provides playback equalisation by using frequency selective negative feedback. The equalised signal is fed to an amplifier via a playback level control which pre-sets the output. This can be useful to match the output level of this circuit with that of another if the equipment being fed has more than one input source.

The mute stage enables the sound to be muted during certain times, such as fast-forward or rewind, when it is preferable not to hear any output.
Playback head

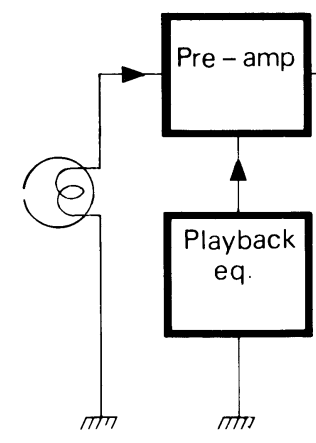

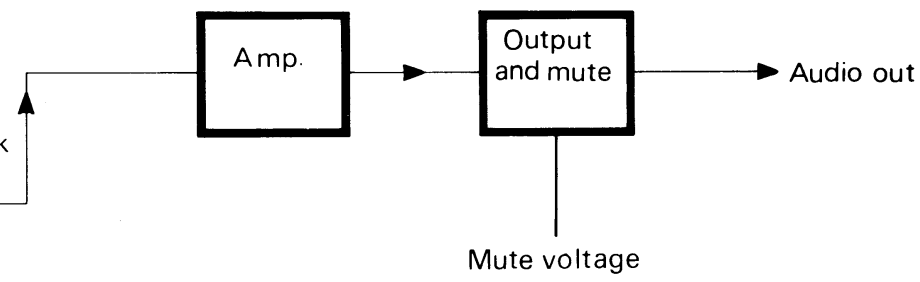

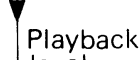
Slevel $\xi$

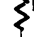
,

तो 